\title{
Aniracetam has proportionately smaller effects on synapses expressing long-term potentiation: Evidence that receptor changes subserve LTP
}

\author{
U. STAUBLI, M. KESSLER, and G. LYNCH \\ University of California, Irvine, California
}

\begin{abstract}
The effects of the nootropic compound aniracetam on synaptic responses expressing long-term potentiation (LTP) were compared with those on control responses in field CA1 of hippocampal slices. The drug produced smaller percentage increases in the amplitude of potentiated, as opposed to control, inputs. Field EPSPs enhanced by the paired-pulse facilitation paradigm, however, were affected to the same degree as control responses. Since aniracetam is known to selectively augment the conductance of AMPA receptor channels, these results indicate that LTP expression is likely to involve modifications of postsynaptic receptors.
\end{abstract}

Long-term potentiation (LTP) is a form of synaptic facilitation with several of the characteristics that are expected for a memory-encoding process (e.g., rapid formation, extreme stability, synapse specificity). Experimental work directed at identifying the sites at which LTP is expressed has focused on three possibilities: (1) an increase in release, (2) a change in the properties of postsynaptic receptors, and (3) a reduction in the resistance of postsynaptic spines, resulting in greater current for a given synaptic conductance. The observation that LTP greatly increases synaptic potentials mediated by the AMPA but not NMDA subclass of glutamate receptors argues strongly against the release hypothesis, since increased release enhances currents generated by both types of receptors (Muller \& Lynch, 1988; see also Kauer, Malenka, \& Nicoll, 1988; Muller, Joly, \& Lynch, 1988). Although recent findings obtained by using variants of quantal analysis (Bekkers \& Stevens, 1990; Malinow \& Tsien, 1990) have been interpreted as implicating increased release in LTP, contradictory results have been

This research was supported by the Air Force Office of Scientific Research, the National Science Foundation, and the National Institute of Health. Correspondence may be addressed to Gary Lynch, Center for the Neurobiology of Learning and Memory, University of California, Irvine, CA 92717. reported (Foster \& McNaughton, in press), and, in any event, the observed effects are open to a variety of explanations in systems (e.g., the hippocampus) where a single axon makes multiple contacts with a target cell (see Lynch \& Baudry, in press). Two predictions arising from the spine-resistance hypothesis (see Wilson, 1984) have not been confirmed experimentally (Jung, Larson, \& Lynch, in press; Larson \& Lynch, in press), which leaves changes in receptors (or the formation of new synapses) as the most likely explanation for LTP.

One strategy used in searching for the substrates of LTP is to test whether or not drugs differentially affect potentiated as opposed to control responses (e.g., Muller, Buchs, Dunant, \& Lynch, 1990). The idea behind this is that if LTP and the drug produce increments (or decrements) in the same variable, the drug will cause smaller percentage effects on potentiated than on control responses. This can be illustrated through the consideration of two cases, both of which follow the usual assumption that the variables involved in synaptic communication (e.g., probability of release, number of transmitter quanta, size of the postsynaptic response to one quantum) operate in a multiplicative fashion (del Castillo \& Katz, 1954). Case 1 considers the situation in which the drug and LTP increment the same step $(X)$ in the release-response sequence, whereas in Case 2 they act on separate steps (X for drug, $\mathrm{Z}$ for $\mathrm{LTP}$ ). 
Control: $\quad R_{0}=X \cdot Y \cdot Z \quad$ Drug effect: $\frac{R_{1}}{R_{0}}=\frac{(X+A) \cdot Y \cdot Z}{X \cdot Y \cdot Z}=\frac{X+A}{X}$

Control + drug: $\mathbf{R}_{\mathbf{1}}=(\mathrm{X}+\mathrm{A}) \cdot \mathbf{Y} \cdot \mathrm{Z}$

\% Effect: $\frac{A}{X} \cdot 100$

Case 1: Drug and potentiation act at the same step

LTP: $\quad R_{2}=(X+B) \cdot Y \cdot Z \quad$ Drug effect: $\frac{R_{3}}{R_{2}}=\frac{(X+A+B) \cdot Y \cdot Z}{(X+B) \cdot Y \cdot Z}=\frac{X+A+B}{X+B}$

LTP + drug: $\quad \mathbf{R}_{\mathbf{3}}=(\mathrm{X}+\mathrm{A}+\mathrm{B}) \cdot \mathrm{Y} \cdot \mathrm{Z}$

\% Effect: $\frac{A}{X+B} \cdot 100$

Case 2: Drug and potentiation act at different steps

LTP: $\quad R_{2}=X \cdot Y \cdot(Z+B) \quad$ Drug effect: $\frac{R_{3}}{R_{2}}=\frac{(X+A) \cdot Y \cdot(Z+B)}{X \cdot Y \cdot(Z+B)}=\frac{X+A}{X}$

LTP + drug: $\quad \mathrm{R}_{3}=(\mathrm{X}+\mathrm{A}) \cdot \mathrm{Y} \cdot(\mathrm{Z}+\mathrm{B})$

\% Effect: $\frac{\mathrm{A}}{\mathrm{X}} \cdot 100$

Percent drug effect on control vs. potentiated responses:

Case 1: $\quad \frac{\mathrm{A}}{\mathrm{X}}>\frac{\mathrm{A}}{\mathrm{X}+\mathrm{B}}$ if $\mathrm{B}>0$ Case 2: $\quad \frac{\mathrm{A}}{\mathrm{X}}=\frac{\mathrm{A}}{\mathrm{X}}$

As is evident, if the drug and LTP produce an increment in the same step in the release-response sequence, the drug will have a smaller percentage effect on potentiated than on control responses. If they act on different steps, the drug should have the same percentage effect on both responses. (A multiplicative effect of either manipulation would also result in the drug's having the same percentage effect on both types of responses.) Accordingly, a reduction in the proportionate effect of a drug on potentiated synapses would implicate the target of the drug in the expression of LTP.

Recently, it has been found that the nootropic compound aniracetam produces a marked increase in the conductance of AMPA receptors, expressed from rat brain mRNA in oocytes, without changing the properties of NMDA, kainate, and GABA receptors. As was expected from this, the drug also augmented synaptic responses in hippocampal slices (Ito, Tanake, Kohda, \& Sugiyama, 1990). LTP, as has been mentioned, is expressed by a selective increase in the synaptic currents generated by AMPA receptors with little or no change in those mediated by NMDA receptors. Here we report that aniracetam has smaller percentage effects on potentiated than on control responses in field CA1 of the hippocampus. We also show that the drug has the same proportional effects on responses transiently facilitated by a presynaptic mechanism as it does on control responses. These results strongly suggest that changes in AMPA receptors account for the expression of LTP.

\section{METHOD}

Slices of hippocampus were prepared from 3-month-old rats and maintained in an interface chamber with constant subfusion, by means of conventional techniques. Stimulation electrodes were placed in fields CA1a and CAlc, so as to activate separate populations of Schaffer-commissural axons, and an extracellular recording pipette was lowered in the middle of the s. radiatum (the dendritic target zone of the Schaffer-commissural projections) of field CA1b. Single pulses were delivered to each stimulation electrode once every $20 \mathrm{sec}(10-\mathrm{sec}$ interval between pulses to each electrode), and the resultant negative-going field EPSPs were recorded and analyzed on line. Recording was continued until a baseline that was stable for both responses over 15-30 min was collected. LTP was then induced in one of the two inputs, using a theta burst stimulation (TBS) pattern (Larson \& Lynch, 1986); in most experiments, three TBS episodes were used in an effort to achieve maximal potentiation (usually $-50 \%$ above baseline). If stable LTP was present, aniracetam at $1.5 \mathrm{mM}$ (a concentration shown to selectively increase AMPA receptor conductance; Ito et al., 1990) was infused into the chamber for 15-25 $\mathrm{min}$, and its effects on the amplitude of potentiated and control field EPSPs were monitored. Twenty experiments were conducted in this manner.

Seven slices were also tested for the effects of aniracetam on the transient response enhancement produced by the paired-pulse facilitation paradigm. Two pulses separated by $50 \mathrm{msec}$ were delivered to a single control electrode, and this was repeated eight times over a 5-min period both before and during drug infusion. The effects of aniracetam were expressed as a percent change in the amplitude of the second (facilitated) responses. 


\section{RESULTS}

Figure 1A illustrates the effects of aniracetam on the amplitude of control and potentiated field EPSPs expressed as a percentage of their predrug averages. The drug effect had a rapid onset $(<10 \mathrm{~min}$ ), elicited its maximum effects in 15-20 min, and produced a smaller percentage increase in the potentiated input than in the control pathway. A second experiment in which paired-pulse facilitation of the control input was also measured before and after infusion of aniracetam is shown in Figure 1B. Note that the drug increased the second (facilitated) response to the same degree as the control response while having a smaller proportional effect on the potentiated input. The results for the group of experiments are summarized in Table 1. Aniracetam increased control field EPSPs by an average of $25 \%$ and potentiated responses by $14 \%$, a difference that was highly significant. The per- centage effects of the drug were not correlated with the absolute amplitude of the responses in either case. In Table 1 , the effects of the drug on control and potentiated responses within individual slices are compared; the percent change for the former is divided into percent change occurring in the latter. As is shown, the effect of the drug on inputs expressing LTP was on the average about one half of that seen on control inputs terminating in the same dendritic region. A similar analysis for field EPSPs enhanced by paired-pulse facilitation is also summarized in Table 1. As is shown, aniracetam increased the facilitated responses to the same degree as it did control responses.

It is important to note that paired-pulse facilitation increased the evoked potentials to about the same degree as did LTP ( $49 \pm 8 \%$ vs. $41 \pm 16 \%$, respectively). Hence the differential effect of the drug on control as opposed to potentiated inputs cannot be ascribed to differences in their relative amplitudes.

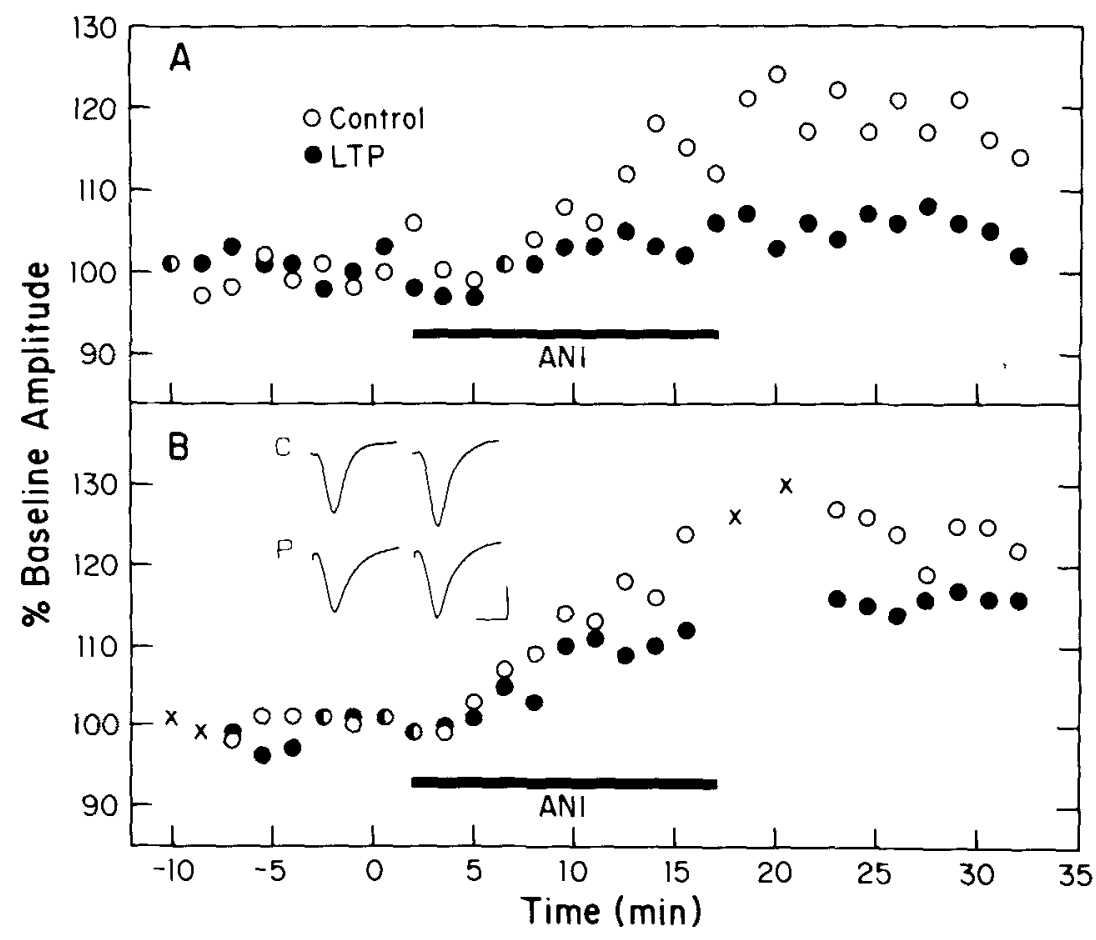

Figure 1. Panel A illustrates a typical experiment done with two stimulating electrodes and a single recording electrode. Long-term potentiation was induced in one of the pathways ( $\bullet 45$ min prior to drug infusion. Each value shown is an average of the amplitudes of four successive field EPSPs and is expressed as a percent of the mean of all responses collected for that pathway during a 10-min predrug baseline. Aniracetam (1.5 mM) was infused into the slice during the period denoted by the horizontal bar. Panel B shows a similar experiment with another slice, except that paired-pulse facilitation was measured at the beginning of the predrug baseline and following a 15-min infusion of $1.5-\mathrm{mM}$ aniracetam. Two sets of four facilitated responses each were collected during a 5-min interval. The mean amplitudes of the second (facilitated) responses in the pairs collected before drug infusion were considered as the baseline and the mean of each separate group of four facilitated responses $(X)$ was expressed as a percentage of this baseline. The inset shows examples of control (C) and potentiated (P) responses before (left) and after (right) infusion of aniracetam. The calibration bars indicate $1 \mathrm{mV}$ and $10 \mathrm{msec}$. 
Table 1

Effects of Aniracetam on Control, Facilitated, and Potentiated Responses $\left(\bar{X}_{ \pm} S D\right)$

LTP (Group Comparison):

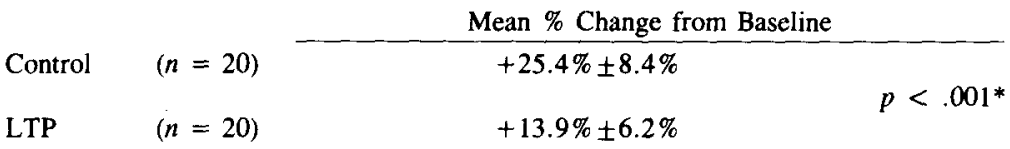

LTP and Paired-Pulse Facilitation (Within-Slice Comparisons):

\begin{tabular}{|c|c|c|c|c|}
\hline \multirow[b]{2}{*}{$\frac{\text { LTP }}{\text { Control }}$} & \multirow[b]{2}{*}{$(n=20)$} & Within-Slice Ratio & \multicolumn{2}{|c|}{$\left(\frac{\% \text { drug effect on experimental response }}{\% \text { drug effect on control response }}\right)$} \\
\hline & & & $0.56 \pm 0.23 \ddagger$ & $p<.001 \dagger$ \\
\hline$\frac{\text { Facilitated }}{\text { Control }}$ & $(n=7)$ & & $1.01 \pm 0.25 \ddagger$ & \\
\hline
\end{tabular}

\section{DISCUSSION}

Several manipulations thought to increase transmitter release (e.g., phorbol esters [Gustafsson, Hwang, \& Wigström, 1988; Muller et al., 1990]; 4-aminopyridine, changes in calcium concentration, paired pulse facilitation [Muller \& Lynch, 1989]) have been shown in previous studies to cause the same percentage increases in pathways exhibiting LTP as they do in control inputs. Moreover, in some of these cases, the manipulation was found to decrease the percent increase in response produced by paired-pulse facilitation (see Muller \& Lynch, 1989, and references therein). The results described here are thus unusual in that the pharmacological manipulation had a smaller proportional effect on LTP but not on paired-pulse facilitation. It should be noted that Ito et al. (1990), in their description of the effects of aniracetam on AMPA receptor conductance, also tested the drug on four potentiated responses but did not obtain a statistically significant decrease in the percentage effect of the drug. The absence of a statistically significant result in their study probably reflects the small sample size and/or the degree and stability of LTP used.

The pattern of results obtained in the present study suggests that aniracetam and LTP independently add to the same variable (i.e., $X+A+B$; see the introduction). The rapid onset of the drug's effects points to an extracellular site of action, while it seems likely that the changes responsible for LTP are due to intracellular modifications. The magnitude of the aniracetam effect was also different $(25 \%)$ from that of LTP $(40 \%-50 \%)$, which would be expected if the two manipulations have distinct effects. Finally, the changes produced by the drug in control and in potentiated responses indicate separate actions on the same variable. That is, if we take a control response as 100 and add $a+50 \%$ effect for LTP and $a+25 \%$ effect for aniracetam, we obtain a $+17 \%(175 / 150)$ drug effect on potentiated inputs; this is reasonably close to the $14 \%$ increase observed in the experiments. It might be noted that the $40 \%-50 \%$ LTP observed in the present work is typical of extracellular recording studies of field EPSPs in the CA1 subregion of hippocampal slices (see, e.g., Barrionuevo \& Brown, 1983; Kelso \& Brown, 1986).

In summary, the present results show that a drug known to selectively affect the properties of AMPA receptors causes a smaller percentage change in potentiated than in control responses. This finding provides new evidence that LTP is expressed via a change in glutamate receptors.

\section{REFERENCES}

Barrionuevo, G., \& Brown, T. H. (1983). Associative long-term potentiation in hippocampal slices. Proceedings of the National Academy of Sciences, 80, 7347-7351.

Bekkers, J. M., \& Stevens, C. F. (1990). Presynaptic mechanism for long-term potentiation in the hippocampus. Nature, 346, 724-729. del Castillo, J., \& KaTZ, B. (1954). Quantal components of the endplate potential. Journal of Physiology, 124, 560-573.

Foster, T. C., \& MCNaughton, B. L. (in press). Long-term synaptic enhancement in $\mathrm{CAl}$ is due to increased quantal size, not quantal content. Hippocampus.

Gustafsson, H., Huang, Y.-Y., \& Wigström, H. (1988). Phorbol ester-induced synaptic potentiation differs from long-term potentiation in the guinea pig hippocampus in vitro. Neuroscience Letters, 85, 77-81.

Ito, I., Tanabe, S., Kohda, A., \& Sugiyama, H. (1990). Allosteric potentiation of quisqualate receptors by a nootropic drug aniracetam. Journal of Physiology, 424, 533-543.

JunG, M. W., LARSON, J., \& LYNCH, G. (in press). Evidence that changes in spine neck resistance are not responsible for expression of LTP. Synapse.

Kauer, J. A., Malenka, R. C., \& Nicoll, R. A. (1988). A persistent postsynaptic modification mediates long-term potentiation in the hippocampus. Neuron, 1, 911-917.

Kelso, S. R., \& Brown, T. H. (1986). Differential conditioning of associative synaptic enhancement in hippocampal brain slices. Science, 232, 85-87. 
LaRSON, J., \& LYNCH, G. (1986). Induction of synaptic potentiation in hippocampus by patterned stimulation involves two events. Science, 232, 985-988.

LARSON, J., \& LYNCH, G. (in press). A test of the spine resistance hypothesis for LTP expression. Brain Research.

LYNCH, G., \& BAUDRY, M. (in press). Re-evaluating the constraints on hypotheses regarding LTP expression. Hippocampus.

Malinow, R., \& TsIEN, R. W. (1990). Presynaptic enhancement shown by whole-cell recordings of long-term potentiation in hippocampal slices. Nature, 346, 177-180.

Muller, D., Buchs, P-A., Dunant, Y., \& Lynch, G. (1990). Protein kinase $\mathrm{C}$ activity is not responsible for the expression of longterm potentiation in hippocampus. Proceedings of the National Academy of Sciences, 87, 4073-4077.
MUlLER, D. Joly, M., \& LYNCH, G. (1988). Contributions of quisqualate and NMDA receptors to the induction and expression of LTP. Science, 242, 1694-1697.

MULLER, D., \& LYNCH, G. (1988). Long-term potentiation differentially affects two components of synaptic responses in hippocampus. Proceedings of the National Academy of Sciences, 85, 9346-9350. MULLER, D., \& LYNCH, G. (1989). Evidence that changes in presynaptic calcium currents are not responsible for long-term potentiation in hippocampus. Brain Research, 479, 290-299.

WILSON, C. J. (1984). Passive cable properties of dendritic spines and spiny neurons. Journal of Neuroscience, 4, 281-297.

(Manuscript received October 4, 1990; revision accepted for publication October 10, 1990.) 